\title{
OTIMIZAÇÃO DE UM PROGRAMA KAIZEN EM UMA EMPRESA DO SETOR METAL MECÂNICO
}

\author{
Josimara Santos Cardoso ${ }^{1}$, Cristina Garcia da Luz ${ }^{1}$, André Luiz Emmel Silva ${ }^{2 *}$, Patrícia Paz Silva ${ }^{3}$, Jorge André Ribas Moraes ${ }^{4}$ \\ ${ }^{1}$ Curso de Engenharia de Produção, Universidade de Santa Cruz do Sul, Santa Cruz do Sul-RS, Brasil \\ 2 Departamento de Engenharia, Arquitetura e Ciências Agrárias, Universidade de Santa Cruz do Sul, Santa Cruz do Sul-RS, Brasil \\ ${ }^{2}$ Curso de Administração, Universidade de Santa Cruz do Sul, Santa Cruz do Sul-RS, Brasil \\ ${ }_{4}^{4}$ Programa de Pós-graduação em Tecnologia Ambiental-PPGTA, Universidade de Santa Cruz do Sul, Santa Cruz do Sul-RS, Brasil
}

*E-mail: andresilva@unisc.br

Recebido em: 20/07/2016 Aceito em: 27/09/2016

\section{RESUMO}

Manter um programa ativo de melhoria contínua dentro das organizações é um desafio permanente para os gestores. Após a implantação, muitos programas perdem força e caem em descrédito. Este artigo apresenta o programa Kaizen em uma empresa do setor metal mecânico, tendo como objetivo avaliar as etapas de implantação, as facilidades e dificuldades enfrentadas após a implantação e contribuir com sugestões de melhorias. O escopo do trabalho fundamenta-se na base Kaizen e suas práticas de implantação, focando na mudança cultural e comportamental dos funcionários de chão de fábrica. O resultado mostrou que são grandes as chances de abandonar a ferramenta após o surgimento das primeiras melhorias. Para evitar isso, o comprometimento das lideranças e os resultados obtidos em curto período de tempo são elementos vitais.

Palavras-chave: Kaizen. Melhoria contínua. Empresa metal-mecânica

\section{Introdução}

Para o cumprimento de metas cada vez mais bem desenhadas cresce a exigência por qualidade e produtividade dentro do ambiente produtivo. Metodologias baseadas nos Sistemas Toyota de Produção ganham espaço nesse cenário, principalmente as ferramentas de melhoria contínua. A melhoria contínua está pautada na eliminação de desperdícios, melhoria dos produtos, aumento da produtividade, bem como no uso de soluções que se apoiem na motivação e criatividade dos colaboradores para melhorar a prática de seus processos [1]. Baseia-se na crença de que todos os aspectos de uma operação podem ser continuamente melhorados, partindo do princípio que os envolvidos diretamente em uma operação se constituem nos principais atores capazes de identificar alterações que devem ser realizadas [2].

Um dos pontos principais que merecem atenção na área de manufatura refere-se à melhoria de produtividade dos recursos produtivos cujo interesse é aumentar a eficiência, reduzir custos, aumentar a disponibilidade e reduzir os desperdícios [3]. O comportamento organizacional e a importância pela criação de uma base bem estruturada dentro do ambiente de trabalho passam a fazer parte deste contexto através de treinamentos, maior espaço para funcionários contribuir com ideias e maior reconhecimento da empresa para cada sugestão proposta.
Dentro dessa filosofia, um dos principais conceitos é o Kaizen, comumente definido em japonês como processo de melhoria contínua [4-5], melhorando o trabalho em toda a organização, focando nas operações que não agregam valor, eliminando desperdícios e custos desnecessários [6-7]. Este conceito traz a perspectiva de atuar nas causas de um problema potencial antes mesmo de se notar os seus possíveis efeitos [8]. O Kaizen quando conduzido de modo estruturado, combatendose as reais causas dos problemas, e vinculado a uma visão estratégica de situação futura ideal, torna-se uma ferramenta dinâmica e sustentável para a condução de mudanças nos processos [9].

O presente artigo tem por objetivo avaliar as etapas de implantação de um programa Kaizen em uma indústria metal mecânico, as facilidades e dificuldades enfrentadas após a implantação e contribuir com sugestões de melhorias.

\section{Referencial teórico}

Este capítulo apresenta conteúdo teórico que fornece suporte para o trabalho. Foram abordados assuntos relacionados a essência do sistema Kaizen e sua administração, focando cinco tópicos: cultura de melhoria, auto-desenvolvimento, qualificação, Gemba e Hoshin Kanri. 


\subsection{Essência Kaizen}

O caminho da competitividade se resume na redução de custos de produção, pois independentemente da forma como as pessoas veem fazendo o seu trabalho atual existem outras maneiras de torná-los mais eficazes [10]. Porém, o Kaizen além de focar a redução dos custos e eliminação de atividades que não agregam valor, ele almeja fazer isso a cada dia, ou seja, identificando de forma contínua as oportunidades que podem ser trabalhadas, onde cada dia pode ser melhor do que o dia anterior [11-12]. Kaizen são esforços de melhoria contínua, executados por todos, com foco central na busca pela eliminação dos desperdícios [9], onde nenhum dia pode se passar sem que alguma melhoria tenha ocorrido, seja ela na estrutura da empresa ou no indivíduo. Especialistas no assunto sustentam que o Kaizen ajuda a motivar os trabalhadores e focar sua atenção aos aspectos da operação do negócio, de modo a melhorar a produtividade e qualidade do produto [7].

Kaizen é a palavra japonesa para a melhoria, levando em conta todas as atividades não contratadas e parcialmente contratadas que ocorrem no local de trabalho, para melhorar as operações e o meio ambiente [13]. Grandes empresas ao longo da história têm utilizado os princípios da mudança, eficiência e melhoria contínua dos processos, utilizando o modelo de Kaizen para alcançar o sucesso [14].

\subsection{Administração Kaizen}

A melhoria contínua é um processo cíclico, e a quantidade de projetos não é o mais importante, mas sim o momento de sua ocorrência, sendo que a cada período de tempo, alguma melhoria tenha de fato ocorrida [15]. Aplica-se a partir do uso de metodologias sistemáticas que permitem uma análise rigorosa dos problemas crônicos que afetam os resultados, detectando, assim, suas causas raízes e permitindo o desenvolvimento de planos de ação que rompem com os paradigmas e preconceitos instalados [16].

Muitos projetos de melhoria não atingem bons resultados devido a diversos fatores: gestão inapropriada, definição incorreta do objetivo, falta de comprometimento da equipe, entre outros. É importante, portanto, que o gestor tenha claro as variáveis relativas à implantação do projeto de melhoria e concentre esforços para aumentar a eficácia e efetividade dos mesmos [17]. A generalidade e a simplicidade do Kaizen são tanto a sua fraqueza quanto sua força [13]. O conceito está tão profundamente enraizado na mente dos gestores e trabalhadores que muitas vezes eles nem percebem que estão pensando Kaizen [2]. Estudo realizado por Brunet e New [13] mostrou que as implementações de Programas Kaizen estão longe de ser uniformes e que dentro de cada empresa o Kaizen se adaptou a diferentes condições.
Para que seja possível a existência e aplicação do Kaizen, métodos e ferramentas são necessários para que a mudança aconteça. Porém o mais importante é a mudança em cada líder e trabalhador, visto que sem eles é impossível que o sucesso seja alcançado [18]. No ambiente de trabalho não deve haver diferença na forma de tratamento, pois independente do nível de qualificação todos devem ser qualificados para entender o real interesse do Kaizen, e acima de tudo sentirem-se livres para apontar e sugerir mudanças dentro do ambiente de trabalho [19]. $\mathrm{O}$ autor indica cinco tópicos a serem avaliados no sistema de implantação e manutenção do Kaizen: Cultura de melhoria; Autodesenvolvimento; Qualificação; Gemba; e Hoshin Kanri.

\subsubsection{Cultura de melhoria}

É com base na cultura que os comportamentos dos indivíduos se moldam [20]. Assim, o primeiro passo na busca da melhoria contínua passa pela mudança na cultura de todos dentro do ambiente produtivo, ou seja, na forma como cada trabalhador executa sua atividade. Faz-se necessária uma forte e rigorosa transformação, pois sem ela será impossível que os passos seguintes sejam alcançados [18].

Alguns recentes estudos realizados na forma de agir e pensar dos seres humanos apontam que o comportamento do ser humano é repleto de flutuações e que o maior desafio quando se fala no âmbito de mudança cultural é tentar fazer com que essas flutuações sejam pequenas, porém para isso é preciso controlar e respeitar os fatores organizacionais existentes [21].

Algumas estratégias utilizadas para melhorar o desenvolvimento de uma cultura dentro da empresa baseiam-se em entender a atual situação da empresa, ou seja, como a mesma se encontra fazendo uma auto-avaliação no quesito cultural, assim como identificar e compreender os pontos fracos que podem impedir seu desenvolvimento [22]. A mudança de cultura deve ser um dos itens verificados dentro da melhoria contínua e pode ser alavancada através de trabalhos em equipe, autodisciplina, círculos de qualidade, prática de sugestões individuais ou em grupo.

\subsubsection{Autodesenvolvimento}

Em 1973 Peter Drucker apresentou o conceito "Knowledge Worker" ou "trabalhadores com conhecimento" que vem sendo estudado até os dias de hoje e que defende a ideia que cada trabalhador deve dominar e apresentar vasto conhecimento sobre seu posto de trabalho, pois só assim poderá identificar prováveis problemas. Este conceito pode ser resumido por Fiebig, Correia e Schreiner [23] o qual relatam que os problemas devem ser identificados antes mesmo que estes ocorram. 
O treinamento, neste contexto, tem a função de qualificálos a fazê-los agir de forma inteligente, segura e ágil para lidar com as diferentes situações prováveis de ocorrer em seu ambiente de trabalho. Para que uma equipe esteja apta a contribuir com a resolução de problemas e sugestões de mudanças, é fundamental que esteja treinada. Cabe aos gerentes identificar a orientação a ser passada e a forma mais adequada, ou seja, a forma mais clara e objetiva [24].

\subsubsection{Qualificação}

A qualificação é, na verdade, o resultado do autodesenvolvimento, pois os funcionários de uma empresa uma vez treinados estarão qualificados para desenvolver suas atividades e colocarem em prática as orientações dadas. Conforme pesquisas recentes, países como EUA e alguns europeus chegam a gastar em torno de $1 \%$ do Produto Interno Bruto em qualificação. A grande maioria das empresas já percebeu que a qualificação profissional está diretamente associada à produtividade e qualidade dos produtos e serviços por ela prestados [25].

Para que ocorra o "contínuos learning" (aprendendo sempre) faz se necessário o aumento na troca de informação entre funcionários de mesma empresa [26]. Estando uma equipe qualificada para desenvolver determinada função, esta poderá compartilhar da informação com os demais [18]. Para alcançar Kaizen, a gerência aproveita a experiência coletiva (gestão do conhecimento) de todos os seus colaboradores, valorizando a solução de problemas em conjunto [12]. Kaizen simboliza a mobilização da força de trabalho, proporcionando o principal canal para os funcionários contribuírem com o desenvolvimento de sua empresa [13]. Planejar a utilização de fatores relacionados à implantação de projetos de melhoria contínua aumenta o potencial de sucesso e efetividade dos mesmos [17].

\subsubsection{Gemba}

O Gemba é considerado onde tudo acontece, ou seja, "o chão de fábrica" e deve ser também o local onde todos os problemas devem ser resolvidos, pois é o local onde é possível visualizar, entender e achar as causas para tudo que esteja acontecendo de errado ou que necessita ser melhorado [11]. O verdadeiro sucesso do Kaizen está em fazer do Gemba um lugar comumente visitado [27].

O conceito "de visitar o chão de fábrica" foi criado por Taiicho Ohno, um dos fundadores do Sistema Toyota de Produção e defende as cinco regras, chamadas de regras de ouro [27].

a) Sempre vá até o Gemba quando um problema aparece; b) Acesse o gembutsu "a causa do problema". Se por exemplo uma máquina na produção está quebrada ela é a causa do problema, ou se um lote está fora do padrão ele é a causa do problema naquele momento;

c) Tome medidas temporárias;

d) Encontre a raiz do problema utilizando o método "Por quê?";

e) Padronização: uma vez o problema estando resolvido se faz necessária à padronização para uma permanente solução.

As idas ao Gemba das lideranças permitem uma melhor compreensão do fluxo de valor e ajudam a identificar sistemas de gestão inadequados [27]. Também ajuda a quebrar a rigidez da hierarquia da gestão tradicional, permitindo a livre comunicação entre supervisores e funcionários. E quando realizado de forma rotineira, pode promover um sentimento de respeito, propriedade e orgulho entre os funcionários [27].

\subsubsection{Hoshin Kanri}

O Hoshin Kanri possui origem japonesa e pode ser definido como uma prática de planejamento, implementação e revisão estratégica para alcançar metas definidas em busca da qualidade total dos processos. Ou seja, é o desdobramento da política da qualidade difundida desde a gerência até as equipes de implantação [28].

De forma resumida, seria a união dos três pilares da empresa, onde as metas definidas pela alta gerência são supervisionadas pela média gerencia e executadas por equipes de trabalho, todos alinhados e buscando um único objetivo, neste caso a qualidade do processo [29]. Este sistema de gerenciamento permite o preenchimento da lacuna existente entre as estratégias da empresa e as ferramentas de produção enxuta utilizadas [28].

Algumas adaptações têm sido realizadas no Hoshin Kanri visando seu melhor entendimento e resultado, como o modelo FAIR, desenvolvido por Tennant e Roberts [30]. O modelo FAIR possui relação direta com o ciclo PDCA sendo rodado no nível de planejamento [28]. Hoshin Kanri é um framework de gestão estratégica, que se preocupa com as seguintes tarefas: prover um foco na direção da organização, definindo anualmente algumas poucas prioridades estratégicas $(\mathrm{F})$; alinhar as prioridades estratégicas (A); integrar as prioridades estratégicas no gerenciamento do dia-a-dia (I); e prover uma revisão estruturada do progresso das prioridades estratégicas (R) [30].

O grande diferencial da metodologia Hoshin Kanri está na capacidade de alcançar a integração entre os componentes estratégicos de longo-prazo e a eficácia operacional no curto prazo [31]. Seu princípio é que cada integrante da organização 
deve incorporar dentro de sua rotina uma contribuição para os objetivos globais da mesma [32]. O Hoshin Kanri visa construir, no gerenciamento diário do trabalho, ações consistentes para o alcance de algumas poucas prioridades estratégicas vitais que são, em essência, objetivos de ruptura [31].

\section{Metodologia}

O presente estudo caracteriza-se como um estudo de caso. Tal modalidade é indicada quando se deseja uma maior compreensão sobre os fatos pesquisados [33]. Foi realizado em uma empresa de grande porte do ramo metal-mecânico, localizada na região central do Estado do Rio Grande do Sul, Brasil. Utilizou-se uma abordagem de pesquisa qualitativa com o intuito de compreender como a empresa pesquisada organiza o seu programa Kaizen, pois além das variáveis serem de difícil mensuração, tornou-se necessário captar e entender a interpretação e a opinião das pessoas sobre os fatos envolvidos.

Foi estruturado em cinco fases, conforme a metodologia proposta por Miguel [34]:

Fase 1 - Definição da literatura: Nesta inicial etapa do estudo se fez necessário uma profunda busca bibliográfica em artigos, revistas, livros e sites onde estavam relacionados ao escopo determinado.

Fase 2 - Planejamento do caso: Planejamento inicial de como o estudo pode ser difundido e as etapas necessárias para que isto ocorra. Identificação das possíveis variáveis que podem afetar para o desenvolvimento do trabalho. Neste específico caso se trata da disponibilidade da empresa para fornecer todas as informações necessárias assim como conduzir as visitas de forma a deixar o processo de forma transparente para sequência do estudo.

Fase 3 - Condução de um teste piloto: Realizar um teste a partir do planejamento adotado na fase 2, identificar as dificuldades e realizar os ajustes necessários para elaboração do estudo.

Fase 4 - Coleta de dados: Através de visitas a campo, onde os pesquisadores acompanharam o programa, coletaram informações por meio de entrevistas semiestruturadas, observações, conversas informais e leitura de documentos.

Fase 5 - Análise de dados: Análise de todos os dados, gerando assim um relatório com sugestões possíveis de serem implantadas para a gerência da empresa.

\section{Resultados e discussões}

Conforme os tópicos anteriormente mostrados, se faz necessário explorar alguns fatos importantes dentro do chão de fábrica para que seja possível alcançar o objetivo esperado, neste caso à busca do sucesso do sistema Kaizen. Sendo assim, após uma revisão bibliográfica, analisou-se a realidade da empresa, escopo do trabalho e criação de um plano de ação em busca da melhoria do atual Kaizen.

Fundada em 1995, a empresa em estudo atua no segmento metalúrgico e está situada na região central do estado do Rio Grande do Sul, em uma área construída de $20.000 \mathrm{~m}^{2}$. Especializada na fabricação de roscas transportadoras helicoidais, fornece seus produtos para as maiores fabricantes de máquinas agrícolas do mundo, e conta com aproximadamente 400 colaboradores.

\subsection{O programa Kaizen}

Em 2011 após algumas tentativas sem sucesso de implantação do sistema Kaizen a empresa, auxiliada pelo setor de qualidade, adotou a ideia de registrar as melhorias implantadas pelos colaboradores assim como mensurar a economia que esta geraria, retribuindo ao inventor um prêmio. Em torno de 30 funcionários, ligados a sua maioria às atividades de rotinas administrativas, ficaram responsáveis pela a disseminação dessa nova cultura dentro de seus respectivos setores.

Uma ficha para o registro das melhorias foi distribuída a cada setor no começo do mês. Essa ficha fica com o responsável pelo setor, permitindo o acesso a ela pelos funcionários, quando solicitado. Ao final do mês a ficha é devolvida ao setor de qualidade, o qual avalia as melhorias sugeridas e efetuadas. Essa avaliação se baseia principalmente em ganhos financeiros da mesma. Caso seja selecionada a concorrer à premiação, um registro é realizado em forma de relatório e a mesma é acompanhada no decorrer do ano para verificação de sua continuidade. Se verificado o retrocesso, a melhoria sugerida não concorre mais para premiação. A figura 1 mostra um fluxograma para sistemática adotada pelo programa.

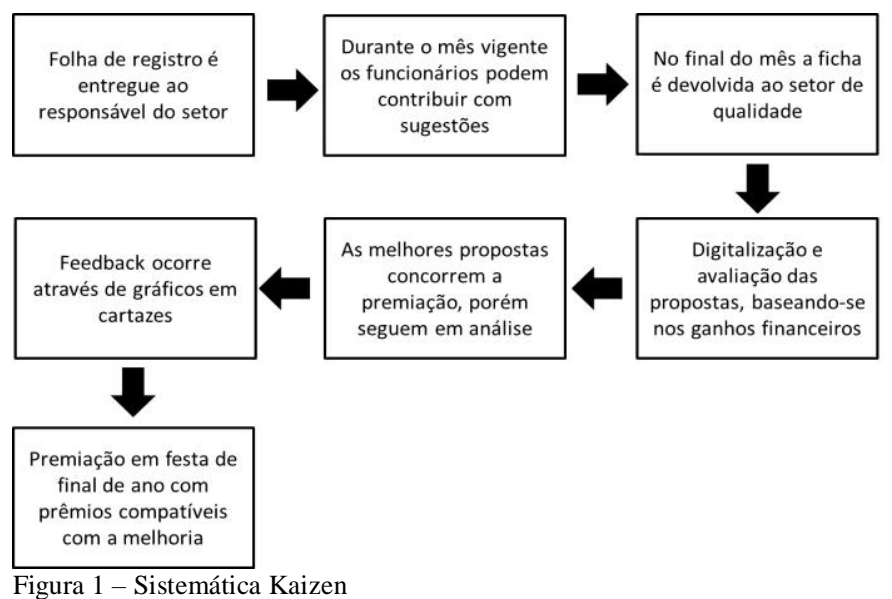


O feedback para os colaboradores ocorre mensalmente através de gráficos que mostram a quantidade total de melhorias implantadas e registradas por setor. Ao final do ano, acontece a premiação na festa da empresa, onde há um reconhecimento perante todos os funcionários e também seus familiares. Os prêmios são compatíveis com o retorno obtido para a empresa com aquela melhoria.

Em 2011 foram 342 melhorias realizadas. Em 2012, 291 e em 2013 o número caiu para 190 melhorias. A maioria está relacionada com adequação de layout; redução de desperdício; melhoria na alocação, identificação e distribuição de componentes; e aumento de produtividade. A queda na quantidade de registros deve-se ao esgotamento das possibilidades de melhorias, ou ao desinteresse pelo programa.

Em entrevista informal e não estruturada com o grupo responsável pelo programa, destacou-se algumas dificuldades e problemas enfrentados pela empresa para a sua manutenção: informalidade; falta de padronização; melhorias caem em desuso; dificuldade na administração das informações, a maioria dos registros está em cópia física, gerando problemas de praticidade; premiação apenas anualmente; e dificuldade de implantação de um sistema de incentivo mensal ou trimestral.

\subsection{Propostas de melhoria para o programa}

Conforme os acompanhamentos realizados foram possíveis visualizar algumas mudanças que se fazem necessárias para que o programa Kaizen continue ativo:

- Ocorrência da premiação em menor espaço de tempo: Diminuir o espaço de tempo entre uma premiação e outra poderia trazer o incentivo motivacional para o dia a dia do funcionário. Em dois meses ou menos, as melhores práticas seriam selecionadas e os responsáveis por sua realização teriam seus nomes, setor e foto expostos em um cartaz de dimensões bem visíveis em algum ambiente da empresa, no refeitório por exemplo. Seria um reconhecimento sem grandes investimentos. A premiação anual não deixaria de existir por propiciar, além do incentivo via bonificação, um estímulo para a continuidade do programa.

- Registro e documentação dos procedimentos: $\mathrm{O}$ registro dos procedimentos, a sistemática dos processos, assim como toda a melhoria alcançada e suas respectivas premiações estão carentes de registros, sendo necessário uma restruturação nos controle e documentos internos. É evidente que a parte de avaliação dos resultados das melhorias não é exata, porém critérios de avaliação, organização e fluxo de documentação assim como formas de registro podem ser padronizados. Isso traria principalmente mais agilidade no processo e independência para empresa no momento de avaliação da melhoria implantada, não dependendo do conhecimento de um funcionário em especifico.

- Software e Terminais (inicialmente caixa de ideias): Com o sistema atual todas as melhorias sugeridas precisam passar pelo responsável do setor, o que toma um tempo desnecessário do mesmo. Começando pela implantação de uma caixa onde os funcionários deixariam suas ideias (em formulário padrão) para serem recolhidas a cada quinzena. Futuramente, implantação de terminais digitais em pontos da empresa onde as sugestões fossem diretamente para um banco de dados, agilizando ainda mais o processo visto que o registro da melhoria seria feito automaticamente, deixando apenas a avaliação para ser realizada posteriormente. Terminais semelhantes a esses já são usados hoje na empresa para as ordens de manutenção. É importante mencionar que tanto a caixa de ideias quanto os terminais para funcionarem requerem $\mathrm{o}$ treinamento do funcionário.

- Treinamento dos funcionários no dia da qualidade anual: O dia da qualidade que ocorre todo ano, onde a produção é interrompida, é uma ótima oportunidade para disseminar a cultura do Kaizen e treinar os funcionários. Treinamento em ferramentas simples, como o "5 porquês", palestras de incentivo e acesso a conteúdo interno como histórico de objetivos alcançados, metas a serem atingidas, valores e princípios (melhoria contínua, responsabilidade, respeito...), exemplos internos a serem seguidos, etc. Outras oficinas e atividades que agregam a instrução do funcionário também poderiam ser desenvolvidas, tudo isso com o intuito de criar uma identidade entre o funcionário e a empresa fazendo com que o mesmo se sinta parte ativa dela.

- Revisão do programa de treinamento para novos funcionários: Novamente se ressalta a importância da criação de uma identidade entre o funcionário e a empresa desde o princípio. O programa de treinamento de novos funcionários precisa visar à difusão da cultura organizacional, seus valores, princípios, o próprio Kaizen e seu funcionamento. Isso afeta diretamente a maneira como o trabalhador encara seu trabalho, de maneira ativa e engajada ou mecânica e desmotivada.

- Treinamento e conscientização da gestão: É vital que as lideranças da empresa incentivem continuamente essa cultura de melhoria. Se o nível mais alto da hierarquia coorporativa não representar aquilo que se busca na organização, todo esforço dos demais poderá falhar. É preciso que as lideranças entendam a importância de se criar uma cultura organizacional e humanizar o trabalho por motivos já citados. Consequentemente é necessário também um treinamento para gestão no sentido de liderar e disseminar essa cultura no dia a dia. Esses treinamentos podem ser oferecidos também no dia anual da qualidade e em outras oportunidades. É importante mencionar que quando lidamos com cultura, lidamos com longo prazo e investimento. Os resultados 
muito provavelmente irão demorar a aparecer, e um investimento considerável, principalmente de tempo, terá de ser feito.

\section{Conclusão}

Depois de feita a avaliação da atual situação da empresa, concluiu-se que a ferramenta Kaizen tem potencial, desde que trabalhada de forma correta, tanto na sua fase de implantação como em sua fase pós-implantação. Este estudo mostrou que as chances de deixar a ferramenta cair em desuso após o surgimento das primeiras melhorias é bastante grande. Para evitar esta tendência é necessário um maior monitoramento e controle de resultados em todo o processo, mantendo sempre todos os funcionários envolvidos atualizados com os resultados do programa.

Outro fator de destaque e que ficou bastante evidente neste estudo foi o quanto importante é o envolvimento da alta administração com o chão de fábrica, ressaltando constantemente a necessidade da melhoria contínua e incentivando os funcionários para que as mudanças ocorram. Isso, quando aliado ao treinamento e qualificação, resulta em colaboradores motivados e capazes de identificar constantes melhorias, não deixando o Kaizen entrar em desuso.

$\mathrm{O}$ alicerce do Kaizen são os funcionários. Estimulando a potencialização de suas virtudes, individuais e coletivas, alcançam-se benefícios em prol da própria organização. A ideia principal é buscar no ser humano o conhecimento para soluções e melhorias para as atividades da rotina. A troca de experiências, o registro e documentação dos procedimentos, o compartilhamento dos resultados e revisões constantes no programa, são fatores importantes para o aprimoramento do Kaizen. Qualquer esforço voltado para esse fim precisa ser incentivado pelas lideranças e alta administração.

\section{IMPROVEMENT OF A KAIZEN PROGRAM INTO A MECHANICAL METAL FACTORY}

ABSTRACT: Keeping a continuous improvement program into de companies is a perpetual challenge for the leaders. After being a program stabilized is usual a powerless period and some of them fall into disrepute. The currently article demonstrates the Kainzen program into a mechanical metal factory and it aims to assess the steps of implantation as well as the facilities and difficulties faced after being the program settle. The scope of the paper is based in techniques and methods of Kaizen and it focus in cultural and behavioral changes of the factory floor. The article results demonstrate the chances of program being abandoned right after the first improvements arise. In order to avoid it the commitment of the leaders and the results acquired in a short period of time are vital.

Keywords: Kaizen, Continuous improvement, Mechanical metal factory

\section{Referências}

[1] GUimarãeS, J. C. F.; SEVERO, E. A.; PEREIRA, A. A.; DORION, E. C. H.; OLEA, P. M.. Inovação no processo e melhoria contínua em uma indústria de plásticos do pólo moveleiro da serra gaúcha. Sistemas \& Gestão, vol. 8, n. 1, p. 34-43, 2013.

[2] IMAI, M. Gemba kaizen: a commonsense, low-cost approach to management. New York, McGraw-Hill. 1997.

[3] CORREA NETTO, O. J. C.; MARINS, E. Melhoria contínua de produtividade no chão de fábrica utilizando metodologia Kaizen - estudo de caso em indústria cosmética. In: SIMPÓSIO DE ADMINISTRAÇÃO DA PRODUÇÃO, LOGÍSTICA E OPERAÇÕES INTERNACIONAIS, 13., 2010, São Paulo. Anais... São Paulo: SIMPOI, 2010.

[4] GLOVER, W. J.; FARRIS, J. A.; AKEN, E. M. V.; DOOLEN,T. L. Critical success factors for the sustainability of Kaizen event human resource outcomes: An empirical study. International Journal of Production Economics, vol. 132, n. 2, p. 197-213, 2011.

[5] TOPUZ, C.; ARASAN, Z. Kaizen-educational: an awareness-raising and motivational enhancement group counseling model. Procedia - Social and Behavioral Sciences, vol. 84, p. 1356-1360, 2013.

[6] YOSHINO, Rui Tadashi. Proposta de um sistema de produção enxuta para o segmento calçadista. 2008. 315 f. Tese (Doutorado em Engenharia de Produção) Escola de Engenharia de São Carlos, Universidade de São Paulo, São Carlos, 2008.

[7] MANO, Y.; AKOTEN, J.; YOSHINO, Y.; SONOBE, T. Teaching Kaizen to small business owners: An experiment in a metalworking cluster in Nairóbi. Journal of the Japanese and International Economies, vol. 33, p. 25-42, 2014.

[8] RITZMAN, L. P.; KRAJEWSKI, L. J. Administração da produção e operações. $8^{\text {a }}$ ed. São Paulo, Prentice Hall, 2008.

[9] ARAUJO, C. A. C.; RENTES, A. F. A metodologia Kaizen na condução de processos de mudança em sistemas de produção enxuta. Revista Gestão Industrial, vol. 2, n. 2, p. 126-135, 2006.

[10] DUCATTI, A. P. S.; LUIZ, J. V. R.; LUIZ, O. R.; SOUZA, F. B Contribuições da teoria das restrições para os processos de melhoria contínua: uma análise teórico-conceitual. In: SIMPÓSIO DE ENGENHARIA DE PRODUÇÃO, 20., 2013, Bauru. Anais... Bauru: UNESP, 2013.

[11] RAHANI, A.R.; AL-ASHRAF, M. Production Flow Analysis through Value Stream Mapping: A Lean Manufacturing Process Case Study. Procedia Engineering, vol. 41, p. 1727-1734, 2012.

[12] MACHADO, C. M. L.; KIPPER, L. M.; FROZZA, R. A proposal for a computational tool with the lean approach to support minimization of waste production process. International Journal of Management, vol. 4, n. 5, p. 78-91, 2013

[13] BRUNET, A. P.; NEW, S. Kaizen in Japan: an empirical study. International Journal of Operations \& Production Management, vol. 23, n. 2, p. 1426-1446, 2003 .

[14] TETTEH, H. A. Kaizen: A Process Improvement Model for the Business of Health Care and Perioperative Nursing Professionals. AORN Journal, vol. 95, n. 1, p. 104-108, 2012. 
[15] SLACK, N. et al. Gerenciamento de Operações e Processos: princípios e práticas de impacto estratégico. $2^{\mathrm{a}}$ ed. Porto Alegre, Bookman, 2013.

[16] GOMES NETO, B. J.; PEREIRA, F. L. G.; MARIANO, S. R. H. Melhoria dos serviços de TI através da aplicação de um modelo de governança e ferramentas de qualidade: um estudo de caso. Sistemas \& Gestão, vol. 7, n. 4, p. 546-553, 2012.

[17] TANAKA, W. Y.; MUNIZ JR., J.; NETO, A. F. Fatores críticos para implantação de projetos de melhoria contínua segundo líderes e consultores industriais. Sistemas \& Gestão, vol. 7, n. 1, p. 103-121, 2012.

[18] TONIN, P. O. Conceito kaizen como dispositivo de otimização gerencial em sistemas produtivos. In: XVIII SIMPÓSIO DE ENGENHARIA DE PRODUÇÃO, 18., 2011, Bauru. Anais... Bauru: UNESP, 2011.

[19] DOMBROWSKI, U., MIELKE, T. Lean Leadership - fundamental principles and their application. Procedia CIRP, vol. 7; p. 569-574, 2013.

[20] SCHRÖER, K. M.; TEIXEIRA, E. B.; CASARIN, V. A. Cultura organizacional e a relação do nível de satisfação dos trabalhadores. Espacios. vol. 36, n. 16, 2015.

[21] SHI, G; SHIICHIRO, I. Study on the strategies for developing a safety culture in Industrial Organizations. Procedia Engineering, vol. 43, p. 535-541, 2012.

[22] Oliveira, F. C.; ROOS, C.; PALADINI, E. P. Pós-kaizen como diferencial estratégico: modelo proposto a partir de um estudo de caso numa linha de produção de aeronaves. In: SIMPÓSIO DE ENGENHARIA DE PRODUÇÃO, 16., 2009, Bauru. Anais... Bauru: UNESP, 2009.

[23] FIEBIG, G. O.; CORREIA, A. R.; SCHREINER, F. Análise dos fatores mais impactantes para a obtenção de melhores resultados durante a aplicação dos eventos de melhoria contínua em ambiente de engenharia. In: XIX SIMPOSIO DE ENGENHARIA DE PRODUÇÃO, 19., 2012, Bauru. Anais... Bauru: UNESP, 2012.

[24] MURUTA, R. Transforming knowledge workers into innovation workers to improve corporate productivity. Knowledge-Based Systems, vol. 30, p. 35-47, 2012.

[25] MOURÃO, L. Oportunidades de qualificação profissional no Brasil: reflexões a partir de um panorama quantitativo. Revista de Administração Contemporânea, vol. 13, n. 1, p. 136-153. 2009

[26] HOLTSKOG, H. Continuous Improvement beyond the Lean understanding. Procedia CIRP, vol. 7, p. $575-579,2013$

[27] LIEBENGOOD, S. M.; COOPER, M.; NAGY, P. Going to the Gemba: Identifying Opportunities for Improvement in Radiology. Journal of the American College of Radiology, vol. 10, n. 12, p. 977-979, 2013.

[28] AYALA, N. F.; FRANK, A. G. Modelo de implantação do Hoshin Kanri para o desdobramento estratégico na produção enxuta. In: SIMPÓSIO DE ENGENHARIA DE PRODUÇÃO, 19., 2012, Bauru. Anais... Bauru: UNESP, 2012

[29] TURRIONI, J. B.; SOUSA, L. G. M.; NETO, P. L. O. C. Hoshin Kanri: Uma análise da implementação em operações de manufatura no Brasil. In: XIX ENCONTRO NACIONAL DE ENGENHARIA DE PRODUÇÃO, 19., 1999, Rio de Janeiro. Anais... Rio de Janeiro: ABEPRO, 1999.

[30] TENNANT, C.; ROBERTS, P. Hoshin kanri: implementing the catchball process. Long Range Planning, vol. 34, n. 3, p. 287-308, 2001.

[31] SILVEIRA, G. W.; LIMA, P. E. Inserindo o Hoshin Kanri na agenda de gestão estratégica do desempenho. In: ENCONTRO NACIONAL DE ENGENHARIA DE PRODUÇÃO, 22., 2012, Bento Gonçalves. Anais... Bento Gonçalves: ABEPRO, 2012.
[32] WITCHER, B. J.; CHAU, V. S. Balanced scorecard and Hoshin Kanri: dynamic capabilities for managing strategic fit. Management Decision, vol. 45, $\mathrm{n}$. 3 , p. 518-538, 2007.

[33] YIN, R. K. Estudo de Caso: planejamento e método. $4^{\mathrm{a}}$ ed. Porto Alegre, Bookman, 2010

[34] MIGUEL, C. A. P. Metodologia de Pesquisa em Engenharia de Produção e Gestão de Operações. $2^{\mathrm{a}}$ ed. Rio de Janeiro, Elsevier, 2011. 